\title{
Pulsatile MHD Arterial Blood Flow in the Presence of Double Stenoses
}

\author{
M. K. Sharma ${ }^{1 \dagger}$, P. R. Sharma ${ }^{2}$ and V. Nasha ${ }^{3}$ \\ ${ }^{1,3}$ Department of Mathematics, Guru Jambheshwar University of Science \& Technology, Hisar (Haryana) 125001 \\ India \\ ${ }^{2}$ Department of Mathematics, University of Rajasthan, Jaipur (Rajasthan) 302004 , India
}

†Corresponding Author Email: drms123@gmail.com

(Received March 22, 2011; accepted February 1, 2013)

\begin{abstract}
The hemodynamics provides a way to predict effect of atherosclerosis by means of mathematical models. The pulsatile flow of blood through an artery with two side-to-side axisymmetric stenoses has been considered. A static transverse magnetic field to the flow is taken into account. The velocity profile, Wall Shear Stress and Wall Shear Stress Gradient to the flow have been simulated under the influence of magnetic field for various values of length and thickness of the stenosis. The upstream flow velocity in the subsequent stenosis region is significantly lower down from the velocity in the preceding stenosis region. The flow velocity decreases with the increase of Hartmann number. In the stenosis region wall shear stress (WSS) increases from unstenosed region to maximum thickness of stenosis. The wall shear stress (WSS) increases with the increase of Hartmann number and Womersley number. The WSSG have local maximum value in the vicinity of the throat of the stenoses and oscillates in the stenosed portion of the artery. The magnitude of WSSG is directly proportional to the Hartmann number. WSSG increases in magnitude on the upstream and downstream section of both the stenoses with the increase of Womersley number. Generated data are analyzed and discussed through graphs.
\end{abstract}

Keywords: Stenosed artery, Unsteady flow, Newtonian incompressible fluid, Hartmann number, Wall shear stress, Wall shear stress gradient.

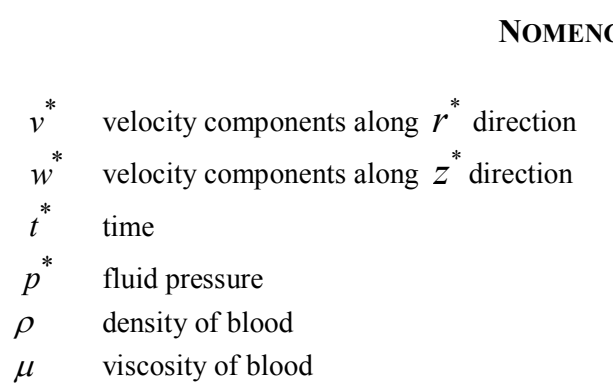

\section{INTRODUCTION}

Blood is regarded as a suspension of small cells in plasma. So, when blood flows through tubes, due to deposition of fatty substances formation of stenosis occurs. The detailed studies of flow field in a stenosed tube help us to understand arterial diseases. In view of this, several studies of fluid dynamics through stenosed artery have been carried out to evaluate the flow pattern, shear stress and shear stress gradient at the walls under steady and pulsatile flow conditions. Young and Tasi (1973-I, II) considered flow of blood dealing with blood as a Newtonian fluid under steady and unsteady flows across a stenosis. Chaturani and

$\begin{array}{ll}\sigma^{*} & \text { electrical conductivity of blood } \\ B_{0}^{*} & \text { magnetic field strength } \\ \alpha & \text { Womersley parameter } \\ \gamma & \text { amplitude parameter } \\ \omega & \text { frequency } \\ \nu & \text { kinematic viscosity of blood }\end{array}$

Upadhya (1979) studied a two fluid model for blood flow through small diameter tubes. Above authors considered the two fluid model (consists of a core suspension of red cells), and a peripheral red cell free plasma layer. The core has been considered as a couple stress fluids and the plasma layer as a Newtonian fluid. It was observed that effective viscosity increases with tube radius. Haldar (1987) presented oscillatory flow of blood in a stenosed artery to obtained numerical solutions for instantaneous flow rate, resistive impedance, wall shear stress and phase lag. Haldar and Ghosh (1994) studied the effect of an externally applied homogeneous magnetic field on the flow characteristics of blood in a single constricted blood vessel and 
simulated the effect of magnetic field on the pressure gradient, velocity profile and wall shear stress. Sanyal and Maji (1999) discussed the unsteady blood flow through an indented tube with atherosclerosis to investigate the oscillatory flow characteristics of blood in a single constricted blood vessel. Steady axisymmetric flow in a constricted rigid tube and flow pattern with the distribution of pressure and shear stress at the wall were computed using finite difference scheme by Pontrelli (2001). Srivastava (2002) considered particulate suspension blood flow through stenotic arteries, to analyze the effects of hematocrit and stenosis shape and observed that the flow resistance decreases with increasing shape parameter but increases with hematocrit. $\mathrm{Ku}$ et al. (1985) and Bonert et al. (2003) found that low and oscillatory wall shear be 3 . positively correlated to localized intimal thickening of arterial wall. Misra and Shit (2006) studied the blood flow through the arteries in a pathological state taking blood as non-Newtonion fluid and used HerschelBulkley equation for flow.

The motivation of present problem is quantitative assessment of flow and mass transfer in mild stenoses is of great importance, since such stenoses represent the early stage of disease development. Several studies have examined flow through single stenosed artery, but there are only few studies in multiple stenoses. Prasad et al. (2008) studied the flow of Herschel-Bulkley fluid through an inclined tube of non-uniform cross-section with multiple stenoses and observed that flow resistance increases with the heights of stenoses decreases with inclination. Misra et al. (2008) 4 formulated a mathematical model for the flow of blood through a multi-stenosed artery with blood as twolayered fluid. Verma and Parihar (2009) discussed the effects of magnetic field and hematocrit on blood flow in an artery with multiple stenosis. They observed that rise in systolic pressure and fall in diastolic pressure are very harmful for weak heart. Layek et al. (2009) worked on the numerical solution of the unsteady viscous flow in the neighborhood of an overlapping constriction under laminar flow conditions and found that the recirculation regions are formed in the downstream of the overlapping constriction.

Kelvin et al. (2010) worked out on modelling of blood flow resistance for an atherosclerotic artery with multiple stenoses and poststenotic dilatations and observed that variation in resistance to flow ratio are subjected to alterations in flow behaviour index, structural variations in relation to magnitude of vessel stenosis and multiple abnormal segments.

\section{Formulation Of The Problem}

The geometry of the present model consists of an artery of circular cross section with two side-by-side axisymmetric stenoses of impermeable wall. The motivation of considering this sort of geometry is experimental and physiological findings near the branching of artery where atherosclerosis lesion generally occurs.

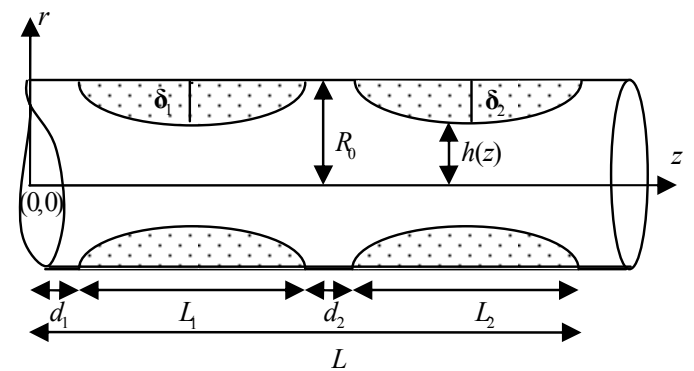

Fig. 1. Physical model of the problem

The present model is first level approximation of the realistic situation where stenoses are taken axisymmetric and situated in a line. A transverse magnetic field of constant strength $B_{0}$ is applied. The calibrating parameters to define double stenosed arterial blood flow are length of stenoses $L_{1}$ and $L_{2}$, thickness of stenoses $\delta_{1}$ and $\delta_{2}$. The boundaries of the stenoses are taken smooth so that it can be approximated in cosinosoidal form. The artery is taken as a straight vessel along the axis of $\mathrm{z}$ (See physical model). Under these considerations, the governing equations of motion of blood through artery with double stenosed in the presence of magnetic field in cylindrical coordinate system $\left(r^{*}, \theta^{*}, z^{*}\right)$ are given by

Equation of continuity

$\frac{1}{r} \frac{\partial\left(r u^{*}\right)}{\partial r^{*}}+\frac{\partial w^{*}}{\partial z^{*}}=0$

The governing equation of motion

$$
\begin{aligned}
& \rho\left(\frac{\partial w^{*}}{\partial t^{*}}+v^{*} \frac{\partial w^{*}}{\partial r^{*}}+w^{*} \frac{\partial w^{*}}{\partial z^{*}}\right) \\
& =-\frac{\partial p^{*}}{\partial z^{*}}+\mu\left(\frac{\partial^{2}}{\partial r^{* 2}}+\frac{1}{r^{*}} \frac{\partial}{\partial r^{*}}+\frac{\partial^{2}}{\partial z^{* 2}}\right) w^{*}-\sigma^{*} B_{0}^{* 2} w^{*}
\end{aligned}
$$

The boundary conditions are

$$
\begin{aligned}
& r^{*}=0: \quad \frac{\partial}{\partial r} w^{*}\left(r^{*}, t^{*}\right)=0 \\
& r^{*}=h^{*}\left(z^{*}\right): \quad w^{*}\left(r^{*}, t^{*}\right)=0
\end{aligned}
$$

\section{Mathematical Analysis}

Using the following non-dimensional parameters

$$
\begin{array}{lr}
r=\frac{r^{*}}{R_{0}}, & z=\frac{z^{*}}{R_{0}}, \\
\frac{\partial p^{*}}{\partial z^{*}}=-q_{0} q(t), & w=\frac{w^{*}}{\left(\frac{q_{0} R_{0}^{2}}{2 \mu}\right)},
\end{array}
$$


M. K. Sharma et al. / JAFM, Vol. 6, No. 3, pp. 331-338, 2013.

$\alpha=R_{0} \sqrt{\frac{\omega^{*}}{v}}, \quad M=\frac{\sigma^{*} B_{0}^{* 2}}{\left(\mu / R_{0}^{2}\right)}$

into the Eq. (2), we get

$\alpha^{2} \frac{\partial w}{\partial t}=2 q(t)+\frac{\partial^{2} w}{\partial r^{2}}+\frac{1}{r} \frac{\partial w}{\partial r}-M w$

Corresponding boundary conditions are reduced to

$$
\begin{array}{ll}
r=0: & \frac{\partial}{\partial r} w(r, t)=0 \\
r=h(z): & w(r, t)=0
\end{array}
$$

where

$$
h(z)=\left\{\begin{array}{cc}
1 & \text { if } 0 \leq z \leq d_{1} \\
1-\frac{\delta_{1}}{2}\left(1+\cos \frac{2 \pi}{L_{1}}\left(z-d_{1}-\frac{L_{1}}{2}\right)\right) & \text { if } d_{1} \leq z \leq d_{1}+L_{1} \\
1 & \text { if } d_{1}+L_{1} \leq z \leq L-L_{2} \\
1-\frac{\delta_{2}}{2}\left(1+\cos \frac{2 \pi}{L_{2}}\left(z-L+\frac{L_{2}}{2}\right)\right) & \text { if } L-L_{2} \leq z \leq L \\
1 & \text { if } L \leq z
\end{array}\right.
$$

Assuming

$q(t)=1+\gamma \cos (\omega t)$

and using into the Eq. (4), we get

$\alpha^{2} \frac{\partial w}{\partial t}=2(1+\gamma \cos (\omega t))+\frac{\partial^{2} w}{\partial r^{2}}+\frac{1}{r} \frac{\partial w}{\partial r}-M w$

Consider,

$w(r, t)=w_{1}(r)+w_{2}(r, t)$

and using into the Eq. (6), the following perturbation equations are obtained

$$
\begin{aligned}
& \frac{1}{r} \frac{\partial}{\partial r}\left(r \frac{\partial w_{1}(r)}{\partial r}\right)-M w_{1}(r)+2=0 \\
& \alpha^{2} \frac{\partial w_{2}(r, t)}{\partial t}=2 \gamma \cos (\omega t))+ \\
& \frac{1}{r} \frac{\partial}{\partial r}\left(r \frac{\partial w_{2}(r, t)}{\partial r}\right)-M w_{2}(r, t)
\end{aligned}
$$

The corresponding boundary conditions are obtained

$$
\begin{array}{lll}
r=0: & \frac{\partial w_{1}(r)}{\partial r}=0, & \frac{\partial w_{2}(r, t)}{\partial r}=0 \\
r=h(z): & w_{1}(r)=0, & w_{2}(r, t)=0
\end{array}
$$

The Eq. (8) is a well known Bessel's differential equation and its solution is given by

$$
w_{1}(r)=c_{1} I_{0}(\sqrt{M} r)+c_{2} K_{0}(\sqrt{M} r)+\frac{2}{M}
$$

where $I_{0}$ and $K_{0}$ are modified Bessel's functions. $c_{1}$ and $c_{2}$ are constants of integration and their values are obtained by applying boundary conditions Eq. (9). Through straight forward calculations the expression of $w_{1}(r)$ is known and given by

$w_{1}(r)=\frac{2}{M}\left[1-\frac{I_{0}(\sqrt{M} r)}{I_{0}(\sqrt{M} h(z))}\right]$

Taking Laplace transform of Eq. (9), we get

$$
\begin{aligned}
& \frac{1}{r} \frac{\partial}{\partial r}\left(r \frac{\partial \bar{w}_{2}(r, s)}{\partial r}\right)- \\
& \quad\left(M+\alpha^{2} s\right) \bar{w}_{2}(r, s)=-\frac{2 \gamma s}{s^{2}+\omega^{2}}
\end{aligned}
$$

The corresponding boundary conditions are reduced to

$$
\begin{array}{ll}
r=0: & \frac{\partial}{\partial r} w_{2}(r, s)=0 \\
r=h(z): & w_{2}(r, s)=0
\end{array}
$$

The Eq. (13) is a non-homogeneous Bessel's differential equation whose solution is given by

$$
\begin{aligned}
& \bar{w}_{2}(r, s)=c_{3} I_{0}(r g(s))+ \\
& c_{4} K_{0}(r g(s))+\frac{2 \gamma s}{\left(s^{2}+\omega^{2}\right) g(s)^{2}}
\end{aligned}
$$

where $g(s)=\sqrt{M+\alpha^{2} s}, c_{3}$ and $c_{4}$ are constant of integration and their values are obtained using boundary conditions Eq. (14). Through straight forward calculation, the expression of $\bar{w}_{2}(r, s)$ in the Laplace domain is known and given by

$$
\bar{w}_{2}(r, s)=\frac{2 \gamma s}{\left(s^{2}+\omega^{2}\right) g(s)^{2}}\left(1-\frac{I_{0}(r g(s))}{I_{0}(g(s) h(z))}\right)
$$

Taking Laplace inversion on both sides of Eq. (16) (Carslaw and Jaeger), we get

$w_{2}(r, t)=\frac{\gamma}{\pi i} \int_{-\infty}^{\infty}\left(\begin{array}{c}\frac{s e^{s t}}{\left(s^{2}+\omega^{2}\right) g(s)^{2}} \\ \left.1-\frac{I_{0}(g(s) r)}{I_{0}(g(s) h(z))}\right)\end{array}\right) d s$

$s= \pm i \omega$ and $s=-\frac{\left(a_{k}^{2}+M\right)}{\alpha^{2}}$ are poles of integrand of the Eq. (17) with $\beta_{k}=a_{k} h(z)$ and $\beta_{k}$ are zeros 
of the Bessel's function of order zero, i.e. $J_{0}\left(\beta_{k}\right)=0 ; k=1,2,3 \ldots$ Applying Cauchy Residue Theorem the solution of $w_{2}(r, t)$ is obtained and hence $w(r, t)=w_{1}(r)+w_{2}(r, t)$

$$
\begin{aligned}
w(r, t) & =\frac{2}{M}\left[1-\frac{I_{0}(r \sqrt{M})}{I_{0}(h(z) \sqrt{M})}\right] \\
+ & {\left[\begin{array}{l}
\frac{\gamma e^{i \omega t}}{A_{1}^{2}}\left(1-\frac{I_{0}\left(A_{1} r\right)}{I_{0}\left(A_{1} h(z)\right)}\right) \\
+\frac{\gamma e^{-i \omega t}}{A_{2}^{2}}\left(1-\frac{I_{0}\left(A_{2} r\right)}{I_{0}\left(A_{2} h(z)\right)}\right) \\
-\sum_{k} \frac{4 \gamma A_{3} e^{-A_{3} t}}{\left(\alpha^{2} A_{3}^{2}+\omega^{2}\right) \beta_{k}}\left(\frac{J_{0}\left(a_{k} r\right)}{J_{1}\left(\beta_{k}\right)}\right)
\end{array}\right] }
\end{aligned}
$$

where

$$
\begin{aligned}
& A_{1}=\sqrt{\left(M+\alpha^{2} i \omega\right)}, \quad A_{2}=\sqrt{\left(M-\alpha^{2} i \omega\right)} \\
& A_{3}=\frac{\left(a_{k}^{2}+M\right)}{\alpha^{2}}
\end{aligned}
$$

\subsection{Volumetric flow rate}

$$
\mathrm{Q}=\frac{2 \pi}{\mathrm{T}} \int_{0}^{\mathrm{T}} \int_{0}^{\mathrm{r}} \mathrm{rw}(\mathrm{r}, \mathrm{t}) \mathrm{dr} \mathrm{dt}
$$

$w(r, t)$ involves complicated expression in the form of Bessel's function therefore its integration has been obtained numerically by using Simpson's one-third rule for double integration and then flow rate profiles are given in Fig. 17.

\subsection{Shear Stress}

The shear stress in dimensionless form is defined as

$$
\begin{aligned}
\tau=\frac{d w}{d r} & =-\frac{2}{\sqrt{M}}\left[\frac{I_{1}(r \sqrt{M})}{I_{0}(h(z) \sqrt{M})}\right] \\
& {\left[\begin{array}{l}
\frac{\gamma e^{i \omega t}}{A_{1}}\left(\frac{I_{1}\left(A_{1} r\right)}{I_{0}\left(A_{1} h(z)\right)}\right) \\
+\frac{\gamma e^{-i \omega t}}{A_{2}}\left(\frac{I_{1}\left(A_{2} r\right)}{I_{0}\left(A_{2} h(z)\right)}\right) \\
-\sum_{k} \frac{4 \gamma A_{3} e^{-A_{3} t}}{\left(\alpha^{2} A_{3}{ }^{2}+\omega^{2}\right) h(z)}\left(\frac{J_{1}\left(a_{k} r\right)}{J_{1}\left(a_{k} h(z)\right)}\right)
\end{array}\right] }
\end{aligned}
$$

\subsection{Wall Shear Stress}

Wall Shear Stress $=\left(\frac{d w}{d r}\right)_{r=h(z)}$

\subsection{Wall Shear Stress Gradient}

The wall shear stress gradient is given by

$$
\begin{aligned}
& \text { WSSG }=\left(\frac{d}{d z}\left(\frac{d w}{d r}\right)\right)_{r=h(z)} \\
& =\frac{2\left(I_{1}(h(z) \sqrt{M})\right)^{2}}{\left(I_{0}(h(z) \sqrt{M})\right)^{2}} h^{\prime}(z) \\
& +\gamma h^{\prime}(z)\left[\begin{array}{l}
\frac{e^{i \omega t}\left(I_{1}\left(A_{1} h(z)\right)\right)^{2}}{\left(I_{0}\left(A_{1} h(z)\right)\right)^{2}} \\
+\frac{e^{-i \omega t}\left(I_{1}\left(A_{2} h(z)\right)\right)^{2}}{\left(I_{0}\left(A_{2} h(z)\right)\right)^{2}} \\
\left(\begin{array}{l}
4 A_{3} e^{-A_{3} t} \\
\left(\alpha^{2} A_{3}^{2}+\omega^{2}\right) h(z) \\
-\sum\left(\frac{2}{h(z)}-\frac{a_{k} J_{2}\left(a_{k} h(z)\right)}{\left(J_{1}\left(a_{k} h(z)\right)\right)}\right.
\end{array}\right)
\end{array}\right]
\end{aligned}
$$

\section{RESUltS AND DiscuSSION}

The effect of double stenoses and magnetic field on the flow of the blood and wall shear stresses is analyzed through Figs. 2 to 17 . Figure 2 depict that upstream flow velocity decreases when it proceed from unstenosed region to first and second stenosed region. This indicates that the volume flow will reduce due to stenosis. Also, it is plausible that the upstream flow velocity in the second stenosis is less than the velocity in the first stenosis. In Fig. 3 it is observed that with the increase of length of first stenosis the flow velocity increases even the stenosis thickness remains unchanged. Figure 4 shows the blood velocity decreases along radial direction with the increases in thickness of first stenosis. Figure 5 demonstrates the effect of Hartmann number on the flow profiles. The flow velocity decreases with the increase of Hartmann number. Figure 6 shows velocity profiles of the flow during a cardiac period at the cross-section corresponding to the peaks of the stenoses. The pulsatile nature of the flow is revealed from the Fig. 6 and magnitude of the flow velocity is higher in the circular region while it decreases in first and second stenoses successively. The variation in shear stress radially at the entry of the stenosed region, in the region of first and subsequent stenosis are shown in Fig. 7, which reveals that the shear stress in the subsequent stenosis is less in magnitude to the shear stress at the preceding stenosis. Also, when the stenosis thickness $\delta_{2}$ was taken $60 \%$ of $R_{0}$ the shear stress in the crosssectional plane through the apex of the second stenosis changes its sign. This behaviour has not been observed in $20 \%$ and $40 \%$ case. 


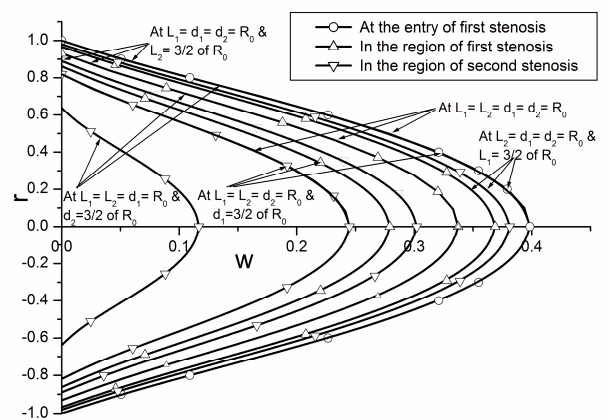

Fig. 2. Variation in velocity profile along radial direction for $\delta_{1}=\delta_{2}=20 \%$ of $R_{0}, \alpha=1.82, \omega t=\pi / 6$

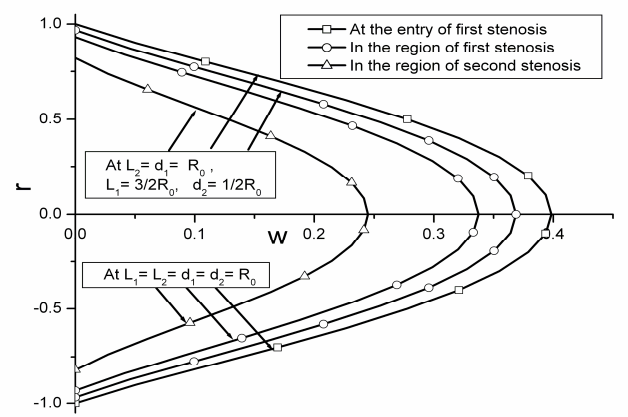

Fig. 3. Variation in velocity versus radial direction for $\delta_{1}=\delta_{2}=20 \%$ of $R_{0}, \alpha=1.82, M=0.5$

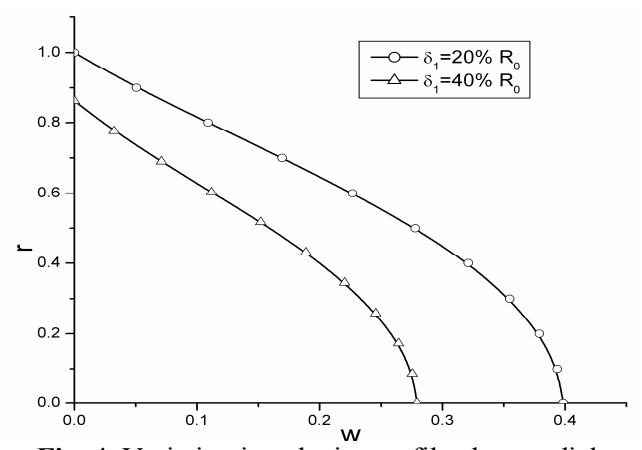

Fig. 4. Variation in velocity profile along radial direction for

$L_{1}=L_{2}=d_{1}=d_{2}=R_{0}, \omega t=\pi / 6, \alpha=1.82, M=0.5$

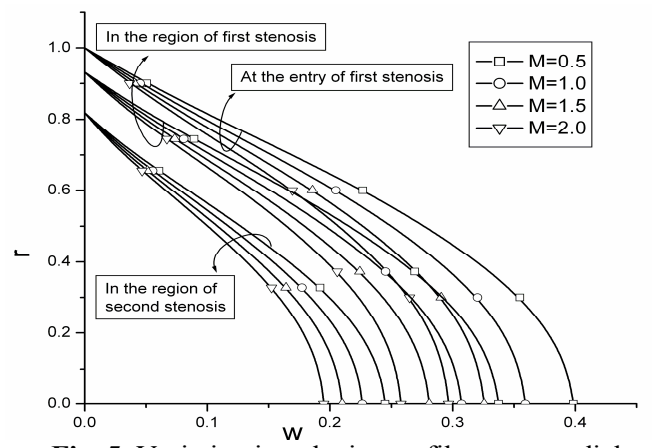

Fig. 5. Variation in velocity profile versus radial direction for $\delta_{1}=\delta_{2}=20 \%$ of $R_{0}$,

$L_{1}=L_{2}=d_{1}=d_{2}=R_{0}, \alpha=1.82, \omega t=\pi / 6$

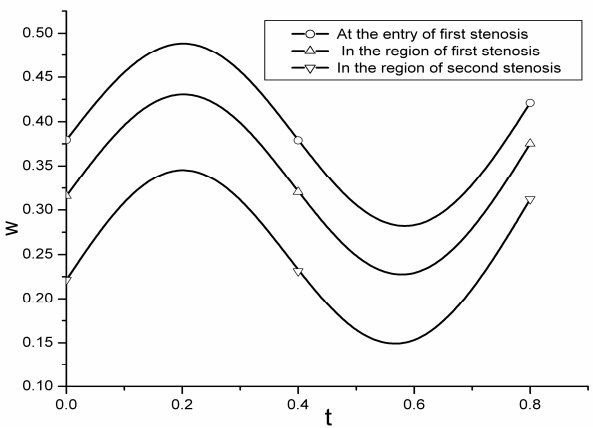

Fig. 6. Variation in velocity versus time for $\alpha=1.82$, $L_{1}=L_{2}=d_{1}=d_{2}=R_{0}, \delta_{1}=\delta_{2}=20 \%$ of $R_{0}, \omega t=\pi / 6$

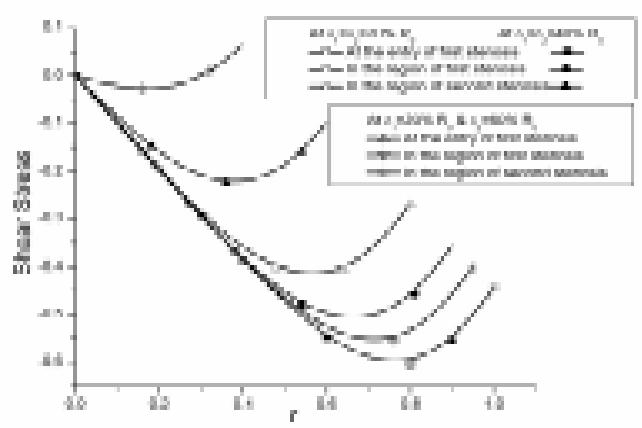

Fig. 7. Variation in shear stress versus radial direction $L_{1}=L_{2}=d_{1}=d_{2}=R_{0}, \omega t=\pi / 6, \alpha=1.82, M=0.5$

Figures 8 and 9 respectively show the variation in shear stress with time $(t)$ at cross-section corresponding to the apex point of the stenoses at $\mathrm{z}=0.186$ and $\mathrm{z}=0.434$. Also, shear stress is of same nature in both the stenoses and at $r=h / 4$ and $r=h / 2$ reveals that shear stress oscillates in the period of cardiac output and for the systolic flow magnitude of shear stress is higher for downstream to second stenosis while this pattern turned into reversal for the diastolic flow. The shear stress changes its sign twice during a cardiac period when the maximum thickness of stenosis is $60 \%$ of $R_{0}$ and Womersley number $\alpha=1.82$. The wall shear stress variation with time $(\mathrm{t})$ plotted in Fig. 10 at the crosssection corresponding to the maximum thickness point of the stenoses, in both, along the upstream and downstream stenosis. The wall shear stress changes its sign during the cardiac period for the case when $\delta_{2}=$ $60 \%$ of $R_{0}$, it reveals that the point of separation will come into existence. In Fig. 11 it is plausible that in the stenosis region wall shear stress (WSS) increases from unstenosed region to maximum thickness depict that the flow rate decreases through this length which is good agreement with the study of Nicolas et al. (2003). The wall shear stress (WSS) increases with the increase of Hartmann number which is in agreement that Lorenz's force caused by transverse magnetic field decelerate the flow velocity leads to increase in WSS. Figure 12 shows the WSSG have local maximum value in the vicinity of the throat of the stenoses and oscillates in the stenosed portion of the artery. It also reveals that the magnitude of the local maxima of WSSG in the second stenosis along the downstream is lesser in compare to 
the first stenosis. It suggests that in case of multiple stenoses the aggregation of particles will be more in the downstream stenosis and the magnitude of WSSG is directly proportional to the Hartmann number. Figure 13 and 14 shows that WSS increases with the increase of Womersley number. Besides it, the difference of WSS on the stenosed region to WSS on the unstenosed region decreases with the increase of Womersley number. The point of separation is arises when $\alpha=1.82$ and maximum thickness of stenosis is $60 \%$ of $R_{0}$. The separation points are confined in the vicinity of the apex of stenoses on the either sides.

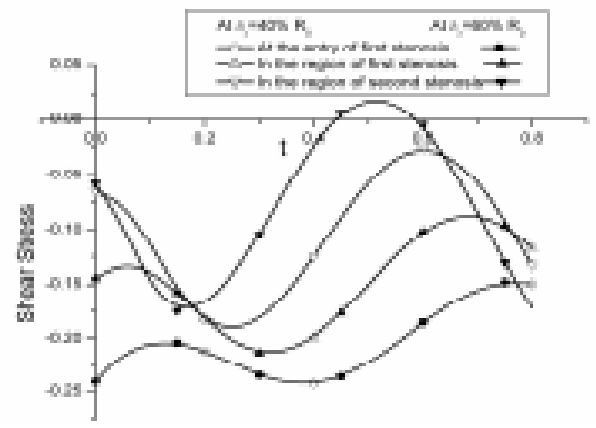

Fig. 8. Variation in shear stress versus time for

$$
r=h / 4
$$

$L_{1}=L_{2}=d_{1}=d_{2}=R_{0}, \omega t=\pi / 6, \alpha=1.82, M=0.5$

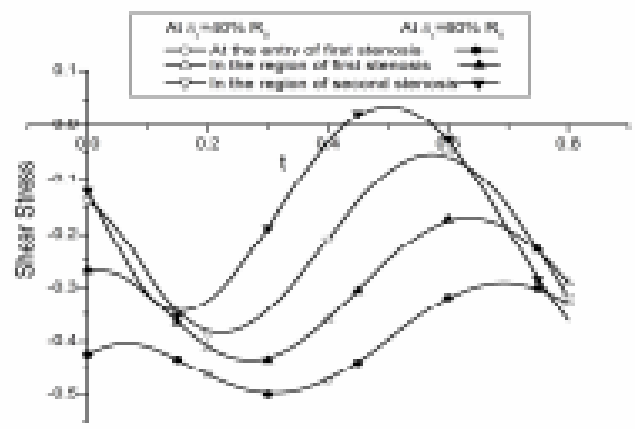

Fig. 9. Variation in shear stress versus time at $r=h / 2, \alpha=1.82$

$\delta_{1}=20 \%$ of $R_{0}, L_{1}=L_{2}=d_{1}=d_{2}=R_{0}, \omega t=\pi / 6$

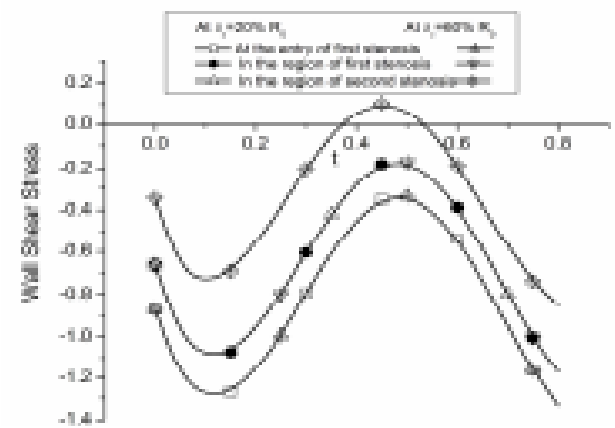

Fig. 10. Variation in WSS versus time for $\delta_{1}=20 \%$ of $R_{0}$,

$L_{1}=L_{2}=d_{1}=d_{2}=R_{0}, \omega t=\pi / 6, \alpha=1.82$

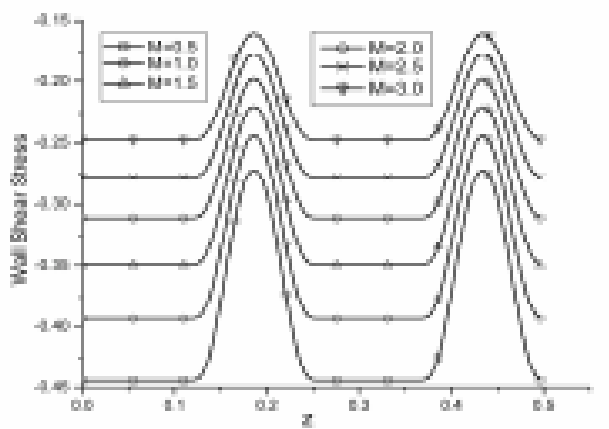

Fig. 11. Variation in wall shear stress versus axial direction for $\delta_{1}=20 \%$ of $R_{0}, L_{1}=L_{2}=d_{1}=d_{2}=R_{0}$,

$$
\alpha=1.82, \omega t=\pi / 6
$$

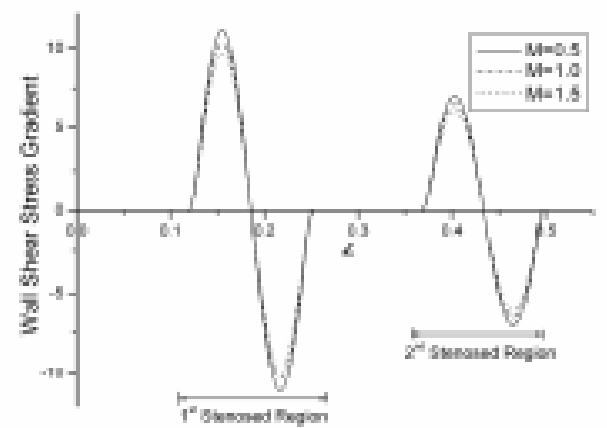

Fig. 12. Variation in wall shear stress versus gradient along axial direction for $L_{1}=L_{2}=d_{1}=d_{2}=R_{0}$,

$$
\alpha=1.82, \delta_{1}=\delta_{2}=20 \% \text { of } R_{0}, \omega t=\pi / 6
$$

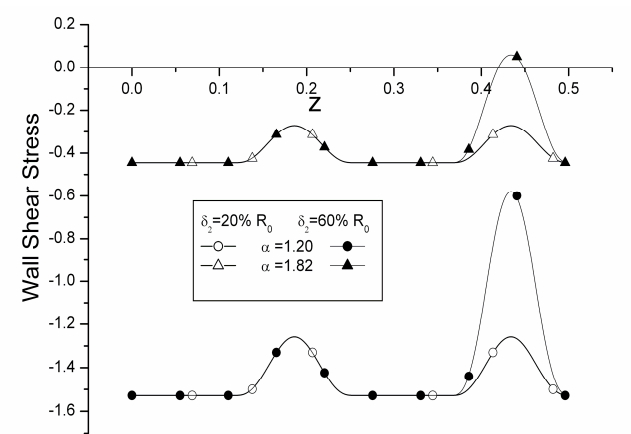

Fig. 13. Variation in wall shear stress along axial direction $L_{1}=L_{2}=d_{1}=d_{2}=R_{0}$, $\delta_{1}=20 \%$ of $R_{0}, \omega t=\pi / 6, M=0.5$

Figure 15 explains the effects of severity of stenosis on the flow behaviour. The separation in the flow arises when maximum thickness of stenosis is $60 \%$ of $R_{0}$. Figure 16 shows the variation of WSSG with Womersley number. It is plausible that WSSG increases in magnitude on the upstream and downstream section of both the stenoses. The magnitude of WSSG on the left stenosis is greater than the stenosis right to it in the flow direction. Figure 17 demonstrates flow rate decreases with the increase of Hartmann number. Also, it depict that flow rate decreases with the increase of Womersley number. 


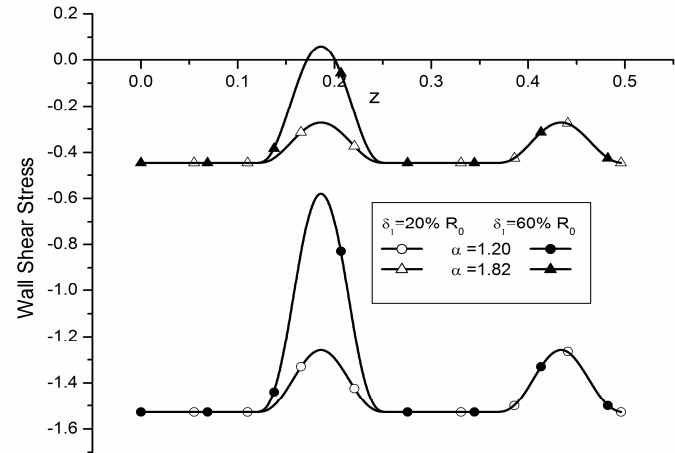

Fig. 14. Variation in wall shear stress along axial direction $L_{1}=L_{2}=d_{1}=d_{2}=R_{0}$, $\delta_{2}=20 \%$ of $R_{0}, \omega t=\pi / 6, M=0.5$

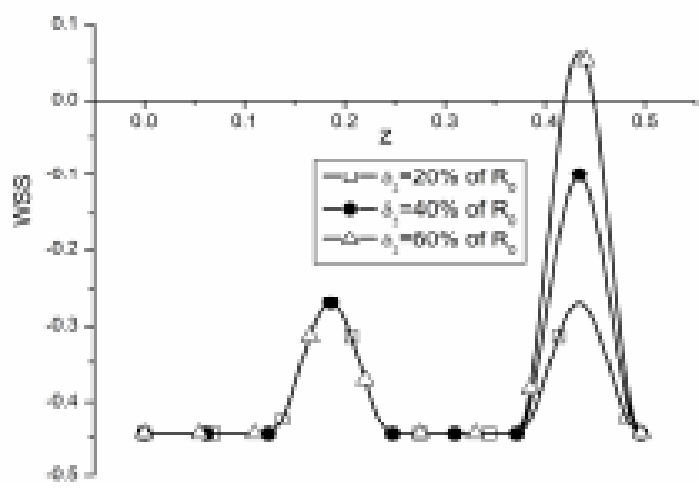

Fig. 15. Variation in wall shear stress along axial direction $L_{1}=L_{2}=d_{1}=d_{2}=R_{0}, \alpha=1.82$ $\delta_{2}=20 \%$ of $R_{0}, \omega t=\pi / 6, M=0.5$

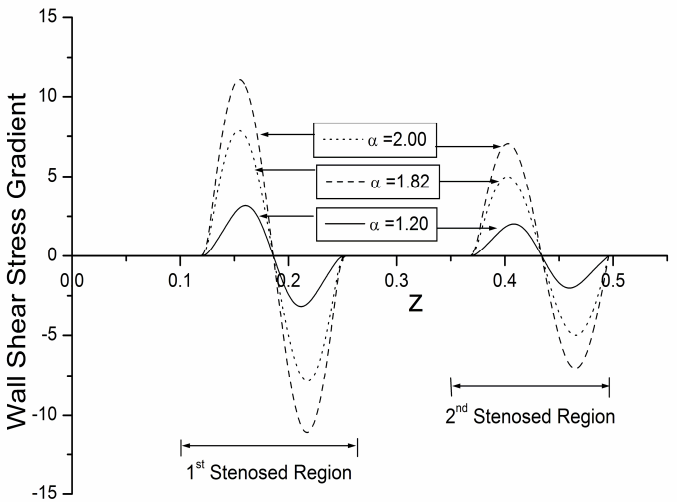

Fig. 16. Variation in wall shear stress gradient along axial direction $L_{1}=L_{2}=d_{1}=d_{2}=R_{0}$, $\delta_{1}=\delta_{2}=20 \%$ of $R_{0}, \omega t=\pi / 6, M=0.5$

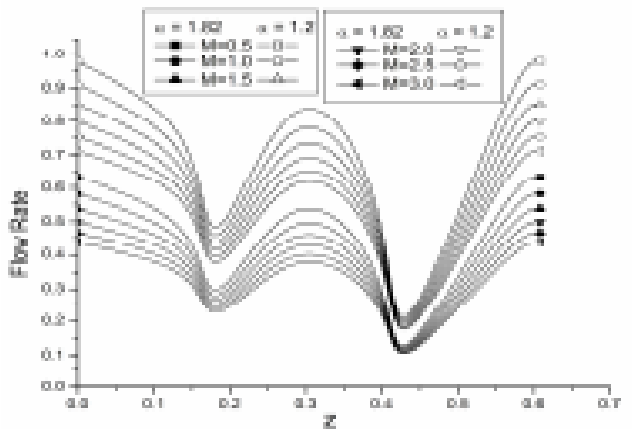

Fig. 17. Variation in flow rate versus axial direction for $\omega t=\pi / 6, \delta_{1}=20 \%$ of $R_{0}$,

$L_{1}=L_{2}=d_{1}=d_{2}=R_{0}, \delta_{2}=40 \%$ of $R_{0}$

\section{CONCLUSION}

(i) The flow velocity is proportional to the length of stenosis even the thickness remains unchanged.

(ii) The transverse magnetic field decelerate the flow velocity leads to increase in WSS.

(iii) The magnitude of WSSG on the left stenosis is greater than the stenosis right to it in the flow direction and directly proportional to the Hartmann number.

(iv) In case of multiple stenoses the aggregation of particles will be more in the downstream stenosis.

(v) The point of separation arises when $\alpha=1.82$ and maximum thickness of stenosis is $60 \%$ of $R_{0}$. The separation points are confined in the vicinity of the apex of stenoses on the either sides.

(vi) The flow rate decreases with the increase of Hartmann number or Womersley number.

\section{REFERENCES}

Batchelor, G. K. (1967), An introduction to fluid dynamics, Cambridge University Press.

Bonert, M., R. L. Leask, J. Butany, C. R. Ethier, J. G. Myers, K. W. Johnston, and M. Ojha (2003). The relationship between wall shear stress distributions and intimal thickening in the human abdominal aorta, Biomed. Eng., Online 2, 18.

Carslaw H. S., and J. S. Jaeger (1968). Operational Methods in Applied Mathematics, Dover Publication, Inc., New York. 
Chaturani, P., and V. S. Upadhya (1979). A two-fluid model for blood flow through small diameter tubes, Biorheology, 16, 109-118.

Haldar, K. (1987). Oscillatory flow of blood in a stenosed artery, Bulletin of Mathematical Biology, 49(3), 279-287.

Haldar, K., and N. K. Ghosh (1994). Effect of a magnetic field on blood flow through an indented tube in the presence of erythrocytes, Indian J. Pure Appl. Math, 25(3), 345-352.

Kelvin Wong, J. Tu, J. Mazumdar, and D. Abbott (2010). Modelling of blood flow resistance for an atherosclerotic artery with multiple stenoses and poststenotic dilatations, Anziam J. 51, C66-C82.

Ku, D. N., D. P. Gidden, C. K. Zarins, and S. Glagov (1985). Pulsatile flow and atherosclerosis in the human carotid bifurcation: positive correlation between plaque location and low oscillating shear stress, Arteniosclerosis 5, 293-302.

Layek, G. C., S. Mukhopadhaya, R. S. R. Gorla (2009). Unsteady viscous flow with variable viscosity in a vascular tube with a double constriction, Int. J. Eng. Sci., 47, 649-659

Lei, M. (1995). Computational fluid dynamics and optimal design of bifurcating blood vessels, Ph.D. Dissertation, Mechanical and Aerospace Engineering Department, Caroline State University, Raleigh, NC.

Long, Q., X. Y. Xu, K. V. Ramnarine, and P. Hoskins (2001). Numerical investigation of physiologically realistic pulsatile flow through arterial stenosis, $J$. Biomech. 2001, 34(10), 1229-1242.

Maruthi Prasad, K., and G. Radhakrishanamacharya (2008). Flow of Herschel-Bulkley fluid through an inclined tube of non-uniform cross-section with multiple stenoses, Arch. Mech., 60(2), 161-172.

Mazumdar, J., K. C. Ang, and L. L. Soh (1991). A mathematical study of non-Newtonian blood flow through elastic arteries. Australas Phys Eng Sci Med., Jun;14(2), 65-73.

Mazumdar, J. N. (1992). Bio-fluid mechanics, World Scientific Press.
McDonald, D. A. (1974). Blood Flow in Arteries, Edward Arnold Ltd., London.

Misra, J. C., and S. Chakravarty (1986). Flow in arteries in the presence of stenosis, J. Biomechanics, 19(11), 707-718.

Misra, J. C., and G. C. Shit (2006). Blood flow through arteries in a pathological state: A theoretical study, International journal of Engineering Science, 44, 662-671.

Misra, J. C., A. Sinha and G. C. Shit (2008). Theoretical analysis of blood flow through an arterial segment having multiple stenosis, J. Mech. Med. Biol., 8, 265 - 279.

Nicolas, B., D. Coisne, E. Donal, and R. Perrault (2003). Experimental study of laminar blood flow through an artery treated by a stent implantation: characterisation of intra-stent wall shear stress, $J$. Biomechanics, 36, 991-998.

Pontrelli, G. (2001). Blood flow through an axisymmetric stenosis, Mech. E. Part H., Journal of Engineering in Medicine, 215(1), 1-10.

Sanyal, D. C., and N. K. Maji (1999). Unsteady blood flow through an indented tube with atherosclerosis, Indian J. Pure Appl. Math, 30(10), 951-959.

Srivastava, V. P. (2002). Particulate suspension blood flow through stenotic arteries: effects of hematocrit and stenosis shape, Indian J. pure appl. Math., 33 (9), 1353-1360.

Verma, N., and R. S. Parihar (2009). Effects of magneto-hydrodynamic and hematocrit on blood flow in an artery with multiple mild stenosis, International Journal of Applied Mathematics and Computation, 1(1), 30-46.

Young, D. F., and F. Y. Tasi (1973). Flow characteristics in models of arterial stenoses-I. Steady flow, J. Biomechanics, 6, 395-410.

Young, D. F., and F. Y. Tasi (1973). Flow characteristics in models of arterial stenoses-II. Unsteady flow, J. Biomechanics, 6, 547-559. 